\title{
Contemporary Management of Non-Appendicular Mucinous and Signet Cell Colorectal Cancer in Community Setting
}

Poornima Sharma, Aung Oakkar, David Hanna, Dorothy Boo, Wendla Citron, Randi Cohen, Russell DeLuca, Karl Kasamon, Yudhishtra Markan, Gayatri Nimmagadda, Mitch OH, Harvinder Singh and Cherif Boutros*

Department of Surgical Oncology, Medical Oncology and Radiation Oncology, Tate Cancer Center, University of Maryland Baltimore Washington Medical Center, Glen Burnie, MD 21061, USA

\begin{abstract}
Introduction: Colon adenocarcinoma, the most common gastrointestinal cancer, has three distinct histological types namely, non-mucinous, mucinous and signet cell type. To date, the clinical implication of mucinous histology is not completely elucidated. Most studies discussing the impact of mucinous pathology in colon cancer are retrospectives analyses from large tertiary centers and report non-concordant results. The aim of our study is to analyze the results of management of non appendicular mucinous and signet colorectal cancer in a community setting.
\end{abstract}

Methods: Under IRB approval, a prospectively maintained database of patients treated for colorectal cancer (1996-2013) at a university-affiliated community cancer center was accessed. Data was collected on excel documents and reported demographic data (age at initial diagnosis, sex), pathological data (site, stage, histological type of cancer) and management data (treatment modalities and survival). Two groups were created: Non mucinous, and mucinous - signet cell colorectal cancer. Differences between groups were analyzed using chi-square, fisher test and Kaplan Meier curves for survival analysis.

Results: 1595 patients were included. Of these, $91.7 \%$ were non-MSCA (non-mucinous signet cell adenocarcinoma), remaining $7.2 \%$ were MCA and $1.1 \%$ was SRCA (signet ring cell carcinoma). Mean age was 67 in the three groups and there was no difference in age distribution. Right colon cancer represented $38 \%$ of non-mucinous colorectal cancer $(n=556)$, and $58 \%(n=77)$ of the mucinous signet cell type. 5 year survival was $92.8 \%, 89.9 \%, 72.3 \%$ and $19.8 \%$ for stage I-IV; respectively for non-mucinous cancer. 5 year survival for mucinous-signet cell was $67.1 \%, 79 \%, 55.4 \%$ and $0 \%$; respectively for stage I-IV; $p<0.05$.

Conclusion: Mucinous and signet cell histology is a clinicopathological distinct type of colorectal cancer that carries a negative impact on patient survival. Despite multimodality management and advance in chemotherapy, stage IV mucinous-signet cell colorectal cancer has a dismal prognosis. New treatment strategies for mucinous-signet cell cancer are needed.

\section{Introduction}

Colorectal cancer is the third most common cancer and second cause of cancer related deaths in the United States. Mucinous adenocarcinoma is a distinct histological subtype that comprises $6-20 \%$ of all colon cancers. Mucinous adenocarcinoma is defined when $>50 \%$ of tumor volume is composed of extracellular mucin. Tumors that have mucinous component that is more than $10 \%$ but less than $50 \%$ are classified as adenocarcinoma with mucinous features. Signet ring carcinoma indicates the presence of intracellular mucin defined by presence of $>50 \%$ signet rings in the pathological specimen and is a rare histological variant accounting for $<1 \%$ of all colorectal carcinomas.

Recent data indicates that mucinous adenocarcinoma and signet cancer of the colon has a distinct genetic profile and clinical presentation compared to non-mucinous histology indicating more aggressive clinical course and poor outcomes with standard therapeutic approach.

Despite advances in the management of colorectal carcinoma, optimal therapeutic approaches have yet to be developed in these histological subtypes.

In an attempt to evaluate our outcomes of patients with advanced colorectal carcinoma based on these histological subtypes, we initiated a retrospective analysis in our community based cancer center to evaluate the clinical characteristics and outcomes of mucinous and signet ring adenocarcinoma (MSCA) as compared to non-mucinous histology.

\section{Materials and Methods}

Using a prospective cancer registry database and with IRB approval, we conducted a retrospective review of data at Tate Cancer Center. We reviewed the data from 1996-2013, reviewing a total of 1595 cancer patients with colorectal cancer that was treated by either surgery and/or with systemic therapy. We identified and reviewed clincopathological information, management, and survival data on patients with advanced mucinous and signet ring adenocarcinoma. The patients with Mucinous and Signet Ring Cell Carcinoma (MSCA) were compared with another cohort of non-mucinous and signet ring adenocarcinoma.

\section{Patients}

Patients with colon carcinoma diagnosed at Tate Cancer Center affiliated with UM BWMC who were diagnosed between 1996 and 2013 were included in analysis. The database of our cancer registry was used to capture information about the age at diagnosis, gender distribution, and stage at presentation, location of tumor, surgical treatment and systemic chemotherapy. Patients with advanced and recurrent MSCA were identified, and we reviewed the medical charts to

*Corresponding author: Boutros C, MD, MSc, FACS, University of Maryland, School of Medicine, Assistant Professor of Surgery, University of Maryland, Baltimore Washington Medical Center, Medical Director, Tate Cancer Center Chief, Surgical Oncology 305 Hospital Drive, Glen Burnie, MD 21061, USA, Tel: 410-553-8150; Fax: 410-553-8135; E-mail: cboutros@bwmc.umms.org

Received February 03, 2015; Accepted April 10, 2015; Published April 17, 2015

Citation: Sharma P, OakkarA, Hanna D, Boo D, Citron W, et al. (2015) Contemporary Management of Non-Appendicular Mucinous and Signet Cell Colorectal Cancer in Community Setting. Surgery Curr Res 5: 228. doi:10.4172/2161-1076.1000228

Copyright: @ 2015 Sharma $P$, et al. This is an open-access article distributed under the terms of the Creative Commons Attribution License, which permits unrestricted use, distribution, and reproduction in any medium, provided the original author and source are credited. 
assess the treatment provided in terms of systemic chemotherapy and their outcomes.

\section{Statistical analysis}

Survival data was analyzed using the Kaplan-Meier. Statistical tests that were performed were 2 -sided, and $P$ values less than 0.05 were considered to be statistically significant. Survival rate was calculated from the date of diagnosis for the primary tumor until death from any cause.

\section{Results}

The cancer registry identified 1595 patients with colon cancer diagnosed between 1996 and 2013. Of these, 91.7\% were non-MSCA, remaining $7.2 \%$ were MCA and $1.1 \%$ was SRCA. Median age of diagnosis of MRCA and non-MRCA was the same at 67 years. The gender distribution was similar between the MAC/signet and nonmucinous histologies as summarized in Table 1. MSCA were more likely to present with Right sided tumors compared to non-mucinous counterparts $(\mathrm{p}<0.005)$

The distribution of the tumor by Stage for MSCA/signet ring vs. Non-mucinous histology is summarized in Table 2. A total of $32 \%$ of patients with non-mucinous histology presented with stage III and IV disease as opposed to $62 \%$ of patients with mucinous/signet ring histology $(\mathrm{p}<0.05)$ (Figure 1).

\section{Median survival for stage IV patients}

1. 5 year survival rate for all stages and histological subtypes is summarized in Table 3. 5 year survival for stage IV non-mucinous histology is $19.8 \%$ compared to $0 \%$ for mucinous/signet cell histology. Charts of all patients with advanced stage mucinous and signet ring were reviewed to assess the chemotherapy regimens employed with overall disease related outcomes. Regimens that were used to treat these patients included a variety of contemporary regimens employing 5-FU, oxaliplatin, irinotecan and biological agents including EGFR inhibitors and VEGF inhibitors similar to non-mucinous counterparts (Figure 2).

\section{Discussion}

Mucinous adenocarcinoma of the colon is a distinct clinicopathological entity. Molecular studies indicate upregulation of genes involved in cell differentiation and mucin metabolism [1]. Clinically mucinous adenocarcinoma of the colon is more frequently located in the proximal colon and is more frequently associated with MSI phenotype [2]

\begin{tabular}{|l|c|c|c|}
\hline & $\begin{array}{c}\text { Non-MSCA } \\
(\mathbf{n = 1 4 6 3 )}\end{array}$ & $\begin{array}{c}\text { MCA }(\mathbf{n = 1 1 4}) \text { and } \\
\text { SRCA }(\mathbf{n = 1 8})\end{array}$ & $\begin{array}{c}\text { Statistical } \\
\text { Significance (p) }\end{array}$ \\
\hline Median age (year) & 67 & 67.5 & NS \\
\hline \multirow{2}{*}{ Location of tumor } & $\begin{array}{c}\text { Right side }(n=556 ; \\
38 \%)\end{array}$ & $\begin{array}{c}\text { Right side }(n=77, \\
58 \%)\end{array}$ & $p<0.0001$ \\
\cline { 2 - 4 } & $\begin{array}{c}\text { Left side }(n=907, \\
62 \%)\end{array}$ & $\begin{array}{c}\text { Left side }(n=54, \\
41.6 \%)\end{array}$ & $p<0.0001$ \\
\cline { 2 - 4 } & Colon Nos=4.2\% & & N/A \\
\hline
\end{tabular}

Table 1: Age of Diagnosis and tumor location.

\begin{tabular}{|c|c|c|c|}
\hline Stage & $\begin{array}{c}\text { Non-MCA/non- } \\
\text { SRCA }\end{array}$ & MCA/SRCA & $\begin{array}{c}\text { Statistical } \\
\text { Significance (p) }\end{array}$ \\
\hline I & $305(20.8 \%)$ & $12(9.1 \%)$ & $\mathrm{p}=0.0011$ \\
\hline II & $345(23.6 \%)$ & $50(37.8 \%)$ & $\mathrm{p}=0.0001$ \\
\hline III & $279(19.1 \%)$ & $51(38.6 \%)$ & $\mathrm{p}<0.0001$ \\
\hline IV & $190(13.0 \%)$ & $19(14.3 \%)$ & $\mathrm{p}=0.2468$ \\
\hline
\end{tabular}

Table 2: Stage Distribution.
The data regarding adjuvant chemotherapy in mucinous adenocarcinoma of the colon is conflicted. Two large retrospective reviews comparing the benefit of adjuvant chemotherapy in stage III colon cancer between mucinous and non-mucinous histologies found comparable benefit between the two groups $[3,4]$. Another smaller study from Korea found lower disease free survival in patients with mucinous adenocarcinoma and adenocarcinoma with mucinous histology compared to non-mucinous histology with FOLFOX chemotherapy [5]. A recently published meta-analysis including 44 studies and 222,256 patients evaluating the prognostic significance of mucinous histology in colon carcinoma concluded that mucinous adenocarcinoma is associated with $2-8 \%$ increased hazard of death which persisted after correction for stage [6].

Data regarding the responsiveness of advanced colon cancer to systemic chemotherapy is summarized below. A study of 135 patients compared the responsiveness of infusional 5-FU as first line treatment of advanced colon cancer between mucinous and non-mucinous colon adenocarcinoma. Inferior response rate and reduced survival was noted for the mucinous adenocarcinoma group [7]. Another study indicated poor responsiveness of mucinous adenocarcinoma to FOLFOX type chemotherapy regimen [8].

A retrospective review of 255 patients of whom 19\% were MAC and $81 \%$ were non-mucinous histology indicated that overall response rates were significantly higher with non-mucinous vs. mucinous histology (19\% vs. 49\%). After median follow up of 45 months, median overall survival was significantly longer in non-mucinous vs. mucinous histology (23.4 vs.14 mths). In multivariate analysis, mucinous histology was associated with overall poor survival with $\mathrm{HR}=1.59$. The chemotherapy regimen employed in this patient population included variety of oxaliplatin, irinotecan and 5-FU based modern chemotherapy programs [4].

Some of the other studies however have concluded fairly similar 5 year survivals between adenocarcinoma and mucinous adenocarcinoma [9]. One study concluded that mucinous adenocarcinomas of the rectum were associated with increased risk of death as opposed to nonmucinous adenocarcinomas [10].

Signet ring histology appears to be uniformly associated with poor clinical outcome based on review of literature with association with high grade, advanced stage at presentation and poor overall survival [9-11]. Our retrospective review of patients with colon cancer indicates that mucinous and signet ring carcinomas tend to present with higher stage compared to non-mucinous carcinomas. Also amongst patients with stage IV disease, patients with mucinous and signet ring histology have significantly worse median survival and dismal 5 year survival rate compared to their non-mucinous counterpart despite being treated at the same facility. We propose that this histological subset should be one of the stratifying factors in randomized clinical trials of colon cancer. Further novel treatment strategies should be employed in the management approach of this disease category.

There are certain drawbacks of this cancer registry based review that are summarized below. The review is retrospective in nature and spans a fairly long time period where therapy for colon cancer has evolved significantly. We also are missing stage information in $23.5 \%$ patients in non-mucinous colon cancer category. We are in the process of improvising and updating our data base. We have also moved to electronic medical records over the last few years and feel that will facilitate data collection process as well.

Review of recent data indicates that mucinous adenocarcinoma 


\section{Survival Probability of Patients with Non-Mucinous Non-Signet, Mucinous, or Signet} Adenocarcinomas: Stage 3

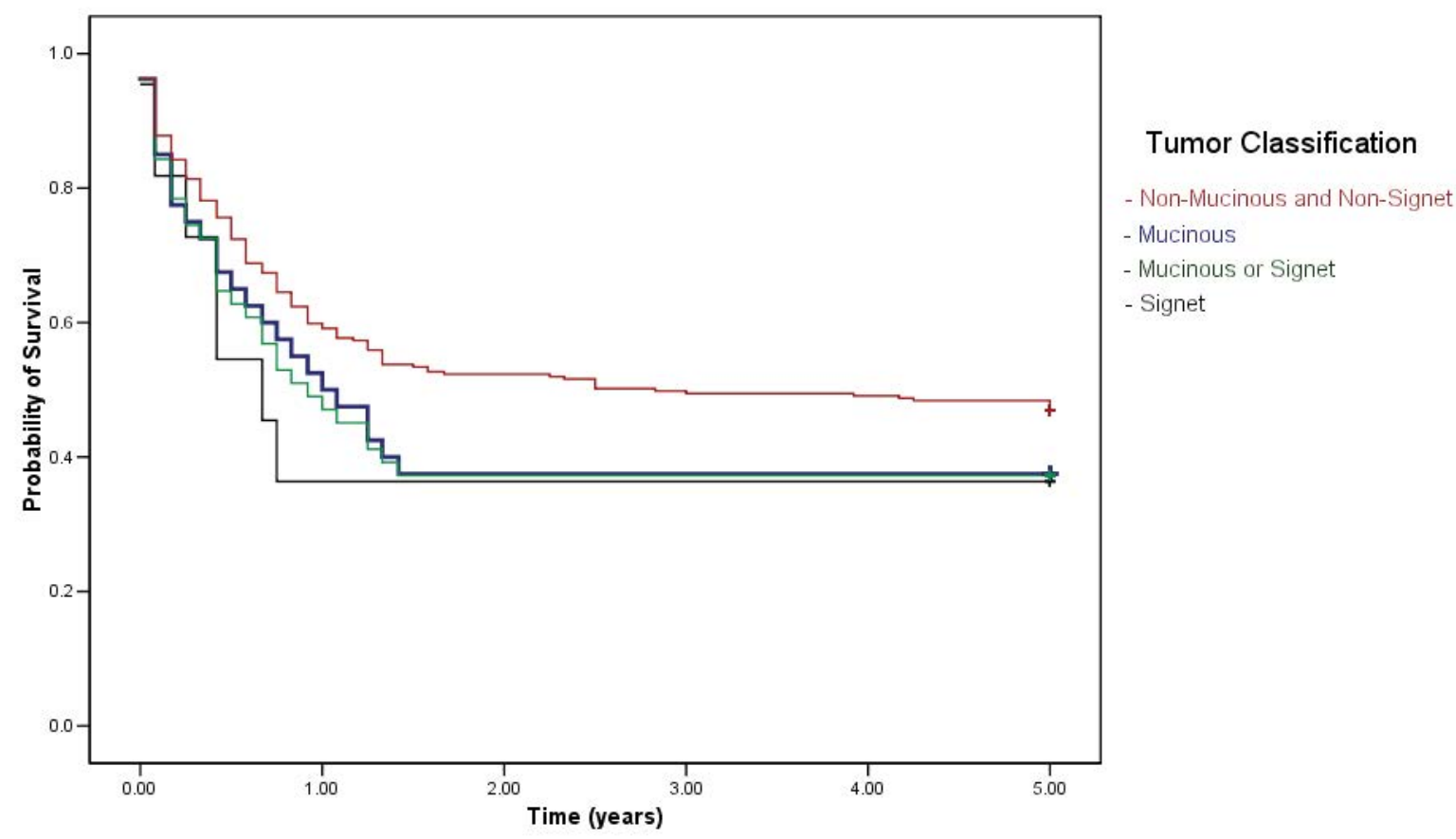

Figure 1: Overall Survival of stage III colorectal adenocarcinoma stratified by tumor mucinous production

Survival Probability of Patients with Non-Mucinous Non-Signet, Mucinous, and Signet Adenocarcinomas: Stage 4

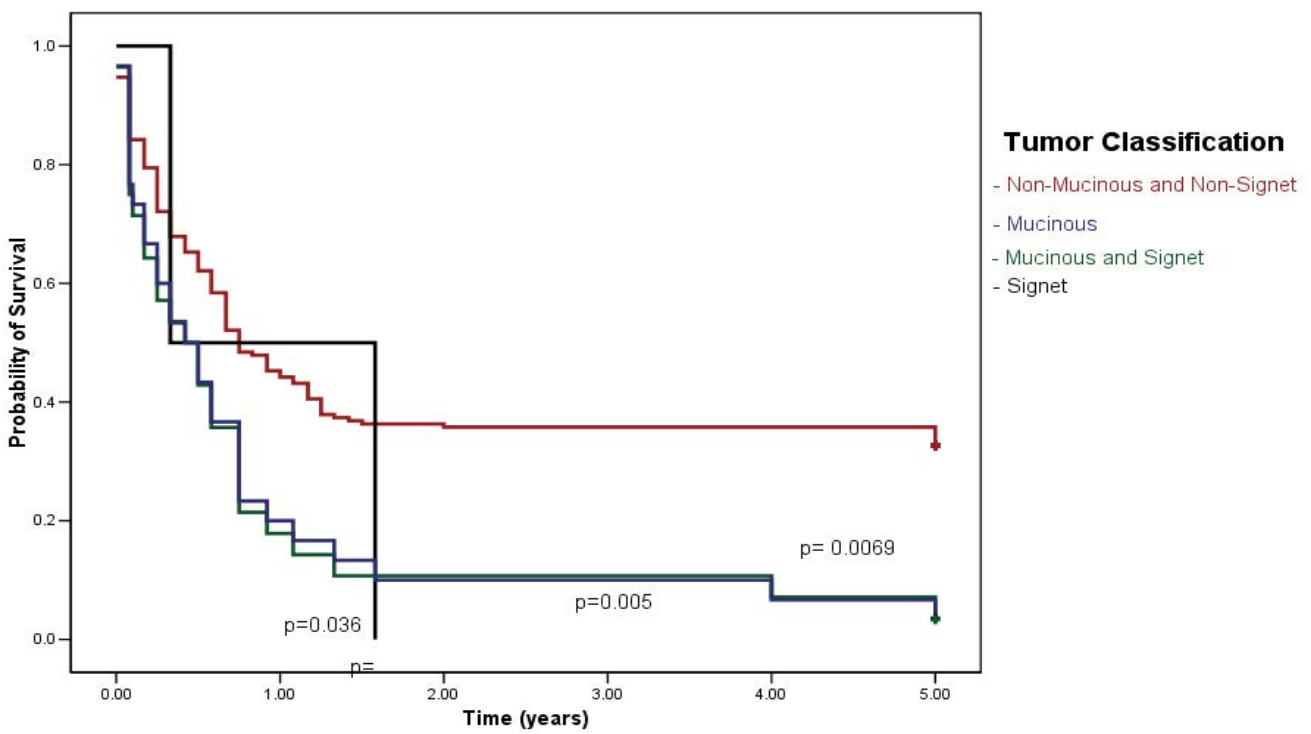

Figure 2: Overall Survival of stage IV colorectal adenocarcinoma stratified by tumor mucinous production 


\begin{tabular}{|c|c|c|c|}
\hline Stage & $\begin{array}{c}\text { Non-MCA/non- } \\
\text { SRCA }\end{array}$ & MCA/SRCA & $\begin{array}{c}\text { Statistical } \\
\text { Significance (p) }\end{array}$ \\
\hline I & $92.8 \%$ & $67.1 \%$ & NS \\
\hline II & $89.9 \%$ & $79 \%$ & NS \\
\hline III & $72.3 \%$ & $55.4 \%$ & NS \\
\hline IV & $19.8 \%$ & $0.0 \%$ & 0.0069 \\
\hline
\end{tabular}

Table 3: 5-Year survival rate

of the colon is associated with poor outcomes when they present with advanced disease and are associated with poor response to systemic chemotherapy. Review of our data from community based cancer center revealed that in in our patient population, advanced colorectal adenocarcinoma had a 5 year survival of $19.8 \%$ with modern chemotherapy programs and multidisciplinary approach. None of the patients with advanced mucinous adenocarcinoma or signet ring cancers survived at 5 years within the same patient cohort. Furthermore, all patients in our data base with signet ring carcinoma presented with advanced disease and with poor outcome making this the worst histological category. This is concordant with several recent publications concluding that signet ring histology presents with advanced stage at diagnosis and have poor clinical outcome [9-11]. We conclude that patients with mucinous adenocarcinoma and signet ring histology should be considered for enrollment in clinical trials with novel agents as they have worse clinical outcome with conventional therapy. One of the pathways that may be targeted in this histological subgroup would be the mucin pathway especially MUC2 and MUC5 proteins using targeted therapy or immunotherapy in combination with chemotherapy and biological agents.

Kang et al. [2] conducted a SEER data base study of colon cancer (1992-2000) and reported that only $10 \%$ of colon adenocarcinoma had mucinous histology and shared similar clinical pictures of the non mucinous colon adenocarcinoma. Globally mucinous adenocarcinoma patient did have worse 5 year overall survival compared to non mucinous adenocarcinoma, however, this was not significant when compared stage for stage. On the other hand the signet cell subtype had clearly the worst prognosis with statistically significant difference than mucinous and non mucinous group of patients. HR of non mucinous adenocarcinoma, mucinous and signet was 1, 1.01, 1.372; respectively. Other important findings from this SEER database study is that mucinous tumors presented more often as advanced stage cancer of the right side of the colon with higher incidence of $\mathrm{LN}$ and peritoneal metastasis.

In a retrospective Asian study including 2006 patients, Song et al. [12] reported close incidence of Mucinous colon adenocarcinoma rate to the western SEER data base study $(7.2 \%$ and $1.2 \%$ Signet cell) The study was also concordant to the western report that mucinous and signet cell adenocarcinoma were associated with high incidence of lymph nodes and peritoneal metastasis. On the other hand, the study did find a statistically significance difference in overall and 5-year survival between non Mucinous, mucinous and signet cell (151 months vs. 67.7 vs. 32.6 ; and $69 \%$ vs., $51 \%$ vs. $27 \%$; respectively)

The relationship of mucinous histology of colon cancer and peritoneal carcinomatosis was also revealed by a large retrospective European study [13]. In this study mucinous histology was found to be an independent factor for synchronous peritoneal carcinomatosis $\mathrm{OR}$ 2.0 (1.6-2.4). In this study median survival of patients with synchronous peritoneal carcinomatosis was only 5 months, much worse than other solid organ metastasis including liver and lung.

Contemporary management of advanced stage colon cancer shows a dramatic improvement in systemic therapy with combination of cytotoxic and biologic agents. This improvement is responsible of a substantial tumor response rate that allows more patients to undergo surgical resection. At the same time multiple non resectional approaches are now available including ablation therapy (RFA, microwave, IRE) and regional therapy (TACE, Y90).

Nozoe et al. [11] previously reported that Mucinous and signet cell histology have difficult clinical behavior. MCs were significantly larger, occurred more frequently in the right and metastasize more often to lymph nodes and to the peritoneal membrane. The 1-, 3-, and 5-year survival rates of patients with MCs were $77.8 \%, 45.4 \%$, and $30.3 \%$, respectively, and were significantly lower than those in patients with NMCs, that were $88.9 \%, 65.6 \%$, and $60.8 \%$, respectively $(\mathrm{P}<0.05)$.

Peritoneal carcinomatiosis of mucinous colon cancer has traditionally being considered an aggressive disease with dismal outcome. Two main factors contribute to this knowledge, poor tumor response to chemotherapy and poor delivery of the systemic chemotherapy to the peritoneal surface. Today, cytoreductive surgery and hyperthermic intraperitoneal chemotherapy has emerged as safe and effective modality of treatment of peritoneal carcinomatosis of colon cancer. The success of this approach in mucinous appendiceal cancer is clear and has been reported in multiple studies making CRS and HIPEC the gold standard for this disease. On the other hand, there is a paucity of reports of results of CRS and HIPEC in colon mucinous and signet cell adenocarcinoma. Studies with relative small number of patients clearly showed that mucinous histology negatively impact the OS of patient undergoing CRS-HIPEC for peritoneal carcinomatosis for metastatic colon cancer [14].

\section{Conclusion}

It is crucial, that contemporary management of advanced stage mucinous and signet cell colon adenocarcinoma is conducted in multidisciplinary approach. While surgery remain an essential component in the management for selected patient, new chemotherapy molecules are needed to increase resectability and to improve survival specially in advanced stages of mucin producing colorectal cancer.

\section{References}

1. Melis M, Yeatman T J, Mcloughlin J M, Siegel E M, Hernandez J, et al. (2010) Gene Expression Profiling of Colorectal Mucinous Adenocarcinomas. Dis Colon Rectum 53: 936-943.

2. Kang H, O'Connell JB, Maggard MA, Sack J, Ko CY (2005) A 10-year outcomes evaluation of mucinous and signet-ring cell carcinoma of the colon and rectum. Dis Colon Rectum 48:1161-1168.

3. Hugen N, Verhoeven RH, Radema SA, H J T De Hingh, Pruijt J F, et al. (2013) Prognosis and value of adjuvant chemotherapy in stage III mucinous colorectal carcinoma. Annals Oncol 24: 2819-2824.

4. Catalano V, Loupakis F, Graziano F, Torresi U, Bisonni R, Mari, et al. (2009) Mucinous histology predicts for poor response rate and overall survival of patients with colorectal cancer and treated with first-line oxaliplatin-and/or irinotecan-based chemotherapy. Br J Cancer 100: 881-887.

5. Kim SH, Roh JK, Shin SJ, Ahn JB, Chung HC, et al. (2013) Prognostic value of mucinous histology depends on microsatellite instability status in patients with stage III colon cancer treated with adjuvant FOLFOX chemotherapy: a retrospective cohort study. Annals Surg Oncol 20: 3407-3413.

6. Verhulst J, Ferdinande L, Demetter P, Ceelen W (2012) Mucinous subtype as prognostic factor in colorectal cancer: a systematic review and meta-analysis. J Clin Pathol 65: 381-388.

7. Negri FV (2005) Mucinous histology predicts for reduced fluorouracil responsiveness and survival in advanced colorectal cancer. Annals Oncol 16: 1305-1310. 
Citation: Sharma P, Oakkar A, Hanna D, Boo D, Citron W, et al. (2015) Contemporary Management of Non-Appendicular Mucinous and Signet Cell Colorectal Cancer in Community Setting. Surgery Curr Res 5: 228. doi:10.4172/2161-1076.1000228

8. Maisano R, Azzarello D, Maisano M, Mafodda A, Bottari M, et al. (2012) Mucinous histology of colon cancer predicts poor outcomes with FOLFOX regimen in metastatic colon cancer. J Chemotherap 24: 212-216.

9. Nitsche U, Rosenberg R, Spath C, Zimmermann A, Bader FG, et al. (2013) Mucinous and signet-ring cell colorectal cancers differ from classical adenocarcinomas in tumor biology and prognosis. Annals Surg 258: 775-783.

10. Hyngstrom JR, Hu C, Xing Y, You YN, Feig BW, et al. (2012) Clinicopathology and outcomes for mucinous and signet ring colorectal adenocarcinoma: analysis from the national cancer data base. Annals Surg Oncol, 19: 28142821.

11. Nozoe T, Anai H, Nasu S, Sugimachi K (2000) Clinicopathological characteristics of mucinous carcinoma of the colon and rectum. J Surg Oncol 75: 103-107.
12. Song W, Wu SJ, He YL, Cai SR, Zhang CH, et al. (2009) Clinicopathologic features and survival of patients with colorectal mucinous, signet-ring cell or non-mucinous adenocarcinoma: experience at an institution in southern China. Chinese Med J 122: 1486-1491.

13. Lemmens VE, Klaver YL, Verwaal VJ, Rutten HJ, Coebergh JWW, et al. (2011) Predictors and survival of synchronous peritoneal carcinomatosis of colorectal origin: A population-based study. Int J Cancer 128: 2717-2725.

14. Yan TD, Chu F, Links M, Kam PC, Glenn D, et al. (2006) Cytoreductive surgery and perioperative intraperitoneal chemotherapy for peritoneal carcinomatosis from colorectal carcinoma: non-mucinous tumour associated with an improved survival. Europ J Surg Oncol 32: 1119-1124. 